\title{
ON LARGE TIME STEP GODUNOV SCHEME FOR HYPERBOLIC CONSERVATION LAWS *
}

\author{
JINGHUA WANG ${ }^{\dagger}$, HAIRUI WEN $\ddagger$, AND TIE ZHOU $\S$
}

\begin{abstract}
In this paper we study the large time step (LTS) Godunov scheme for scalar hyperbolic conservation laws proposed by LeVeque. We show that for an arbitrary Courant number, all the possible wave interactions in each time step occur only in a finite number of cells, and the number of cells is bounded by a constant depending on the Courant number for a given initial value problem. As an application of the result mentioned above, we show that for any given Courant number, if the total variation of the initial value satisfies some conditions, then the numerical solutions of the LTS Godunov scheme will converge to the entropy solutions of the convex scalar conservation laws.
\end{abstract}

Key words. hyperbolic conservation law, Godunov scheme, large time step scheme, entropy condition

AMS(MOS) subject classification: $65 \mathrm{M} 15,35 \mathrm{~L} 65$

\section{Introduction and Notation}

We are concerned with initial value problems for nonlinear hyperbolic conservation laws

$$
\begin{aligned}
& u_{t}+f(u)_{x}=0, \quad-\infty<x<\infty, t \geq 0, \\
& u(x, 0)=u_{0}(x), \quad-\infty<x<\infty,
\end{aligned}
$$

where the flux function $f(u)$ is a smooth function of $u$, and the initial data satisfies

$$
u_{0} \in L^{\infty}(\mathbb{R}) \bigcap B V(\mathbb{R}) \text { with } \quad\left\|u_{0}\right\|_{\infty} \leq M_{0} .
$$

It is well known that solutions to $(1.1)-(1.2)$ generally develop discontinuities even when $u_{0}(x)$ is smooth. Therefore we seek weak solutions, i.e., a locally integrable function $u(x, t)$ satisfying

$$
\iint_{\mathbb{R} \times(0, \infty)}\left[u \varphi_{t}+f(u) \varphi_{x}\right] d x d t+\int_{-\infty}^{\infty} u_{0}(x) \varphi(x, 0) d x=0
$$

for all $\varphi \in C_{0}^{\infty}(\mathbb{R} \times[0, \infty))$.

Moreover, a weak solution $u$ is called an entropy solution, if it satisfies

$$
U(u)_{t}+F(u)_{x} \leq 0
$$

in the sense of distributions, that is

$$
-\iint_{\mathbb{R} \times[0, \infty)}\left[U(u) \varphi_{t}+F(u) \varphi_{x}\right] d x d t \leq 0, \quad \text { for all } 0 \leq \varphi \in C_{0}^{\infty}(\mathbb{R} \times(0, \infty)),
$$

*Received: February 28, 2004; accepted (in revised version): August 14, 2004. Communicated by Tao Tang.

${ }^{\dagger}$ Academy of Mathematics and Systems Science, Academia Sinica, Beijing, 100080, China (jwang@amss.ac.cn). Research supported by National Natural Science Foundation of China (Grant No. 10371124).

$¥$ Academy of Mathematics and Systems Science, Academia Sinica, Beijing, 100080, China (wenhr@amss.ac.cn).

$\S$ LMAM, School of Mathematical Sciences, Peking University, Beijing 100871, China (tzhou@math.pku.edu.cn). Research supported by the China State Major Key Project for Basic Researches (G1999032803). 
where $U$ is any strictly convex function satisfying

$$
0<c_{a} \leq U^{\prime \prime}(u) \leq c_{b}, \quad \text { for }|u| \leq M \text {. }
$$

Here $M$ can be chosen as $u_{0}(-\infty)+T V_{(-\infty, \infty)}\left(u_{0}\right)$ since the LTS Godunov scheme is TVD and does not satisfy maximum principle. (cf.LeVeque[11]). $U$ is called an entropy of problem (1.1)-(1.2); the function $F(u)=\int_{0}^{u} U^{\prime}(\xi) f^{\prime}(\xi) d \xi$ is the associated entropy flux. Any smooth solution $u$ of (1.1) satisfies $U(u)_{t}+F(u)_{x}=0$. The entropy condition (1.4) ensures uniqueness of weak solutions to the initial value problem (1.1)(1.2).

In order to compute the numerical approximation of (1.1)-(1.2), we partition the $x$-axis into intervals of length $h$ by the set of points $x_{i}=i h, i \in \mathbb{Z}$, and the positive time axis into intervals by the points $t_{j}=j \Delta t, j \in \mathbb{N}_{0}$. The grid points $\left(x_{i}, t_{j}\right)$ define a rectangular mesh on $\mathbb{R} \times[0, \infty)$. We will always assume that the time step $\Delta t=\lambda h$ for some fixed mesh ratio $\lambda>0$. We denote the Courant number $C$ as

$$
C=\frac{a \Delta t}{h}, \quad \text { where } a=\sup _{|u| \leq M}\left|f^{\prime}(u)\right| .
$$

Many approximate methods for (1.1)-(1.2) are based on solutions to Riemann problems. At each time step $t=t_{j}$, we just use a piecewise constant function

$$
u_{h}^{j}(x)=u_{i}^{j}, \quad x \in\left[x_{i}, x_{i+1}\right), \quad i=0, \pm 1 \pm 2, \cdots
$$

to approximate the true solution $u\left(x, t_{j}\right)$. Denote the Riemann solution with left and right states $u_{i-1}^{j}$ and $u_{i}^{j}$ at grid point $\left(x_{i}, t_{j}\right)$ as $u_{h, i}^{j}(x, t)$. In convex scalar equations, $u_{h, i}^{j}(x, t)$ is either a shock wave or a rarefaction wave. As long as the Courant number $C<\frac{1}{2}$, the neighboring Riemann solutions will be separated by the intermediate constant states. Therefore, setting

$$
u_{h}^{j}(x, t)=u_{h}^{j}(x)+\sum_{i \in \mathbb{Z}}\left[u_{h, i}^{j}(x, t)-u_{h, i}^{j}\left(x, t_{j}\right)\right]
$$

gives an exact weak solution to (1.1) with initial data $u_{h}^{j}(x)$ in the strip $t_{j}<t<t_{j+1}$. When $C>\frac{1}{2}$ the waves issuing from different grid points may interact with each other and the use of the exact weak solution beyond the time of interaction would be computationally difficult and expensive, except for the Godunov scheme with the Courant number less than or equal to 1 (see [11]). In the large time step (LTS) Godunov scheme proposed by LeVeque [12], the solution (1.7) is taken when $C>\frac{1}{2}$. This means that the waves simply pass through one another with no changes in speed or strength and with no creation of new waves, so they behave as solutions to a linear system $u_{t}+A u_{x}=0$. In other words, we use the linear superposition formula (1.7) to approximate the nonlinear interaction. For convenience, we will still call each wave in (1.7) a shock, admissible discontinuity or rarefaction wave respectively although it is no longer that beyond the linear superpositions.

Although (1.7) will fail to be a weak solution of (1.1) on the strip $t_{j} \leq t<t_{j+1}$ beyond the interaction time, in [11], LeVeque showed that even for an arbitrarily large Courant number, the LTS Godunov scheme gives a consistent approximation for systems of conservation laws and convergent approximation to the initial value problem of (1.1)-(1.2). Wang [15] showed that the LTS Glimm scheme gives a convergent approximation for the Courant number less than or equal to 1 , and a consistent 
approximation for any given Courant number for system of conservation laws. Brenier [2] showed that the LTS Glimm scheme gives a convergent approximation with the Roe Riemann solver for the Courant number less than or equal to 1 . In fact, the approximate solutions constructed by the LTS Godunov and Glimm schemes are a total variation diminishing (TVD) for scalar conservation laws. Other LTS schemes had been introduced by Brenier [1], Toro and Billet [14]. In [11] LeVeque conjectured that his LTS Godunov scheme approximates an entropy solution. As we know, there is not any numerical evidence of entropy violating shocks for these schemes, see [12][8]. Surprisingly, it has been found numerically that the LTS scheme with moderate value of the Courant number (larger than 1 but smaller than 3, say) has much higher resolution, see [12]. In [16] and [17] Wang and Warnecke proved the entropy consistency of the LTS Godunov and Glimm schemes for the Courant numbers less than or equal to 1 for convex scalar conservation laws. If the flux function has constant curvature, the results can be extend to the Courant numbers slightly larger than 1 . And if the flux function is monotone, this holds for the Courant number being 2, and for monotone initial data this is true for an arbitrary Courant number. The entropy consistency of large time step schemes for the isentropic equations of gas dynamics was considered by Jiang and Wang [9]. For $L^{1}(\mathbb{R})$ error estimate of the LTS schemes see [13] and [7]. To our knowledge, the entropy consistency results are essentially proved for the Courant number $C \leq 1$ till now.

In this paper, we will study the LTS Godunov scheme with an arbitrary Courant number. In this case, waves can travel $C$ cells in one time interval, so the linear superpositions of waves cannot be confined in one cell. In section 2 we will prove that in the time interval $\left(t_{j}, t_{j+1}\right)$, all the superpositions are confined in a finite number of cells, and the number is bounded by a constant depending on the Courant number $C$ for a given initial value problem. This means that the total length in $x$ direction of these cells is $O(h)$.

It is well known that if the initial value $u_{0}$ is in $L^{\infty}(\mathbb{R}) \cap B V(\mathbb{R})$, the solutions of the LTS Godunov scheme are bounded uniformly in $L^{\infty}\left(\mathbb{R} \times \mathbb{R}^{+}\right) \cap B V\left(\mathbb{R} \times \mathbb{R}^{+}\right)$; see [11]. Thus the entropy condition (1.5) is a distributional inequality for the signed Radon measure $\eta(u)=U(u)_{t}+F(u)_{x}$. This measure is called the dissipation measure by DiPerna [3]. In section 3, we study the change of the dissipation measure through the linear superpositions. Since the change of the dissipation measure across the linear superposition involving rarefaction waves is too complicated to estimate, we use a piecewise constant function to approximate the rarefaction wave. In the linear superpositions of all kinds of waves, only a shock interacting with a rarefaction wave will increase the dissipation measure. A rarefaction wave meeting another rarefaction wave or a shock interacting another shock will decrease the dissipation measure. We will estimate the net increase amount. The key estimation is (3.11) in Lemma 3.4, which gives a sufficient condition to ensure that the dissipation measure is negative in a linear superposition zone. In section 4 , we prove an entropy consistency theorem (Theorem 4.1) on the entropy consistency for the LTS Godunov scheme. The final result is: for any given Courant number $C$, if the product of increasing total variation and decreasing total variation of the initial data is bounded by a constant depending on the Courant number $C$ for a given initial value problem of convex conservation law, the numerical solutions of the LTS Godunov scheme will converge to the entropy solution, and the constant approaches to infinity as the Courant number tends to $\frac{1}{2}$. 


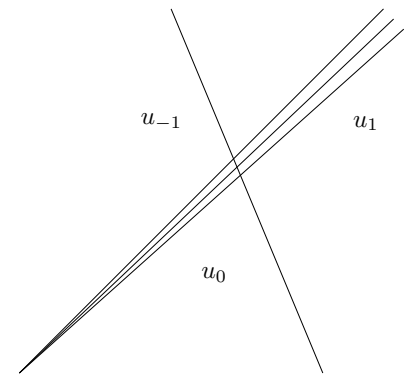

(a) linear superposition of a rarefaction wave with a neighboring admissible discontinuity

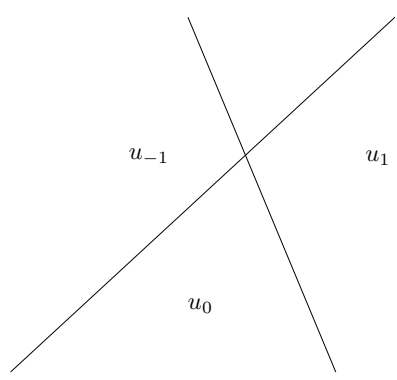

(b) linear superposition of two neighboring admissible discontinuities

FIG. 2.1. Figure for Lemma 2.1

\section{The estimations on linear superposition zone}

In this section, we are concerned with the initial value problem for general scalar conservation law (1.1), (1.2) with the flux function $f$ satisfying

$$
\sup _{|u| \leq M}\left|f^{\prime \prime}(u)\right| \leq c_{2}
$$

where $c_{2}$ is a positive constant.

For the LTS Godunov scheme proposed by LeVeque (1.7), waves issuing from different grid points at time level $t=t_{j}$ may interact(linear superposition) with each other in the time interval $\left(t_{j}, t_{j+1}\right)$. Thus for a group of consecutive waves $\left\{W_{i}\right\}$, if the trajectories of waves in $\left\{W_{i}\right\}$ form a connected set in the strip $t_{j}<t<t_{j+1}$, and if any wave in this group does not interact with waves not in this group, this connected set is actually a connected component. Here and in the sequel, for simplicity, we name trajectories of admissible discontinuities and trajectories of boundaries of rarefaction waves as trajectories of waves. If a closed domain $D$ in the strip $t_{j}<t<t_{j+1}$ is the minimum domain containing a connected component, we call it a linear superposition zone. Specifically, if the group of waves $\left\{W_{i}\right\}$ is

$$
A=\left\{W_{i}, W_{i+1}, \cdots, W_{i+K}\right\},
$$

and the trajectories of these waves form a connected component, then the linear superposition zone $D$ corresponding to A has the following boundaries: the top boundary is the line segment between $\left(x_{i-[C]-1}, t_{j+1}\right)$ and $\left(x_{i+K+[C]+1}, t_{j+1}\right)$, the bottom boundary is the line segment between $\left(x_{i}, t_{j}\right)$ and $\left(x_{i+K}, t_{j}\right)$, the left boundary is a broken line from $\left(x_{i}, t_{j}\right)$ to $\left(x_{i-[C]-1}, t_{j+1}\right)$, and the right boundary is a broken line from $\left(x_{i+K}, t_{j}\right)$ to $\left(x_{i+K+[C]+1}, t_{j+1}\right)$ (see section 3 for the details about the left and the right boundaries).

In Theorem 2.4 we will show that each linear superposition zone contains a finite number of consecutive waves, and the number is bounded by a constant depending on the Courant number for a given initial value problem.

If a pair of neighboring waves interact with each other, we call them an interacting neighbor pair. In each interacting neighbor pair, at least one of the two waves is admissible discontinuity since any two neighboring rarefaction waves cannot interact with each other. 
Lemma 2.1. Let $C$ be the Courant number (defined in (1.6)) for the LTS Godunov scheme (1.7).

(1) If an interacting neighbor pair consists of a rarefaction wave $R\left(u_{-1}, u_{0}\right)$ and an admissible discontinuity $S\left(u_{0}, u_{1}\right)$ (cf. Figure 2.1(a)), then the strength of $S\left(u_{0}, u_{1}\right)$ is bounded below by $\frac{2 a}{c_{2} C}$, i.e.,

$$
\left|u_{0}-u_{1}\right| \geq \frac{2 a}{c_{2} C}
$$

(2) If an interacting neighbor pair consists of two admissible discontinuities $S\left(u_{-1}, u_{0}\right)$ and $S\left(u_{0}, u_{1}\right)$ (cf. Figure 2.1(b)), then,

$$
\left|u_{-1}-u_{1}\right| \geq \frac{2 a}{c_{2} C}
$$

This implies that the strength of at least one of them bounded below by $\frac{a}{c_{2} C}$, i.e.,

$$
\left|u_{-1}-u_{0}\right| \geq \frac{a}{c_{2} C}, \text { or } \quad\left|u_{0}-u_{1}\right| \geq \frac{a}{c_{2} C}
$$

Proof. For (1), since the right boundary of the rarefaction wave $R\left(u_{-1}, u_{0}\right)$ travels with speed $f^{\prime}\left(u_{0}\right)$, and the discontinuity $S\left(u_{0}, u_{1}\right)$ travels with speed $\left(f\left(u_{0}\right)-\right.$ $\left.f\left(u_{1}\right)\right) /\left(u_{0}-u_{1}\right)$

$$
\begin{aligned}
& \left|f^{\prime}\left(u_{0}\right)-\frac{f\left(u_{0}\right)-f\left(u_{1}\right)}{u_{0}-u_{1}}\right| \Delta t \\
= & \left|\frac{f\left(u_{1}\right)-f\left(u_{0}\right)-f^{\prime}\left(u_{0}\right)\left(u_{1}-u_{0}\right)}{u_{0}-u_{1}}\right| \Delta t \\
\leq & \frac{\left(u_{0}-u_{1}\right)^{2} \int_{0}^{1} \xi\left|f^{\prime \prime}\left(\xi u_{0}+(1-\xi) u_{1}\right)\right| d \xi}{\left|u_{0}-u_{1}\right|} \Delta t \\
\leq & \frac{c_{2}}{2}\left|u_{0}-u_{1}\right| \Delta t
\end{aligned}
$$

If

$$
\frac{c_{2}}{2}\left|u_{0}-u_{1}\right| \Delta t<h
$$

then the rarefaction wave (left one) can not catch up the discontinuity (right one) within the time period $0<t<\Delta t$. Hence, in order to let them interact, we must have

$$
\frac{c_{2}}{2}\left|u_{0}-u_{1}\right| \Delta t \geq h
$$

i.e.,

$$
\left|u_{0}-u_{1}\right| \geq \frac{2 a}{c_{2} C}
$$




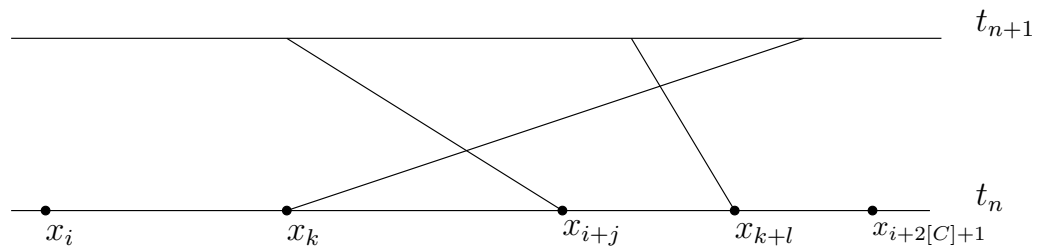

FIG. 2.2. Figure for Lemma 2.2

For (2), since,

$$
\begin{aligned}
& \left|\frac{f\left(u_{-1}\right)-f\left(u_{0}\right)}{u_{-1}-u_{0}}-\frac{f\left(u_{1}\right)-f\left(u_{0}\right)}{u_{1}-u_{0}}\right| \Delta t \\
& \leq \int_{0}^{1}\left|f^{\prime}\left(\xi u_{-1}+(1-\xi) u_{0}\right)-f^{\prime}\left(\xi u_{1}+(1-\xi) u_{0}\right)\right| \xi \Delta t \\
& \leq \int_{0}^{1} \int_{0}^{1}\left|f^{\prime \prime}\left(\eta \xi\left(u_{-1}-u_{1}\right)+\xi u_{-1}+(1-\xi) u_{0}\right)\right|\left|u_{-1}-u_{1}\right| \xi d \eta d \xi \Delta t \\
& \leq c_{2}\left|u_{-1}-u_{1}\right| \int_{0}^{1} \int_{0}^{1} \xi d \eta d \xi \Delta t \\
& \leq \frac{c_{2}}{2}\left|u_{-1}-u_{1}\right| \Delta t
\end{aligned}
$$

by a similar way, we must have

$$
\left|u_{-1}-u_{1}\right| \geq \frac{2 a}{c_{2} C}
$$

to ensure the interaction of the two discontinuities. If $u_{-1}, u_{0}$ and $u_{1}$ are three monotone numbers, then the total strength of the two discontinuities is equal to $\left|u_{-1}-u_{1}\right|$. If $u_{0}$ is not between $u_{-1}$ and $u_{1}$, then

$$
\left|u_{-1}-u_{1}\right|=|| u_{-1}-u_{0}|-| u_{1}-u_{0}||
$$

So the strength of at least one of the discontinuities is greater than $\frac{a}{c_{2} C}$.

Lemma 2.2. If the trajectories of a set of waves $\left\{W_{i}, \cdots, W_{i+p}\right\}(p \geq 1)$ form a connected set in the strip $t_{n}<t<t_{n+1}$, then there exists at least one interacting neighbor pair in this set of waves. And trajectories of $2[C]+2$ consecutive waves in a linear superposition zone contain at least one connected set formed by trajectories of different waves.

Proof: It is easy to know that at least two waves in $\left\{W_{i}, \cdots, W_{i+p}\right\}(p \geq 1)$ must interact with each other. Without loss of generality, we suppose that trajectories of wave $W_{k}$ and trajectories of wave $W_{k+l}$ form a connected $\operatorname{set}(i \leq k, k+l \leq i+p)$, such that $l$ is the smallest positive integer (cf.Figure 2.2). The proof of the first statement is completed if $l=1$. Otherwise, if $l>1$, we have another wave $W_{i+j}$ between $W_{k}$ and $W_{k+l}$, it must interact with either $W_{k}$ or $W_{k+l}$. This contradicts with the fact that $l$ is the smallest.

Now we prove the second statement, suppose that trajectories of waves in $\left\{W_{i}, \cdots, W_{i+2[c]+1}\right\}($ denoted by $A$ ) are contained in a linear superposition $D$, and 
there exists no connected set formed by different waves in $A$, i.e., any two waves in $A$ do not interact with each other in the strip $t_{n}<t<t_{n+1}$. Since $\left\{W_{i-j}\right\}(j \leq 1)$ in $D$ and $\left\{w_{i+k}\right\}(k \geq 2[c]+2)$ in $D$ cannot interact with each other, the trajectories of waves in $D$ would be located on the left side and right side respectively of an interval $(x \prime, x \prime \prime) \subset\left(x_{i}, x_{i+2[c]+1}\right)$. This contradicts the fact that $D$ is a linear superposition zone.

Remark 2.3. From the first statement of Lemma 2.2, there is at least one interacting neighbor pair in a linear superposition zone.

Denote the total variation of a function $u(x)$ over $(a, b)$ by $T V_{(a, b)}(u)$. We have the following important theorem on the size of the linear superposition zone.

Theorem 2.4. Let $C$ be the Courant number (defined in (1.6)) for the LTS Godunov scheme (1.7). Then, a linear superposition zone $D$ contains a finite number of consecutive waves $\left\{W_{i}, W_{i+1}, \cdots, W_{i+K}\right\}, i \in \mathbb{Z}, K \in \mathbb{N}$, and the positive integer $K$ satisfies

$$
K \leq \frac{2 c_{2} C(2 C+1)}{a} T V_{(-\infty, \infty)}\left(u_{0}\right) .
$$

Proof: Denote the set of waves $\left\{W_{i}\right\}$ by $A$ and from the definition of the linear superposition zone, we know that trajectories of waves in $A$ form a connected component. We first show that $K$ must be a finite number. If $D$ contains infinite consecutive waves, we can find subsets $B_{k}$ of $A$ with

$$
B_{k}=\left\{W_{i_{k}}, \cdots, W_{i_{k}+2[C]+1}\right\}, \quad i_{k+1} \geq i_{k}+2[C]+1 .
$$

By Lemma 2.2, there is at least one interacting neighboring pair in each $B_{k}$. By Lemma 2.1, we have

$$
\frac{a}{c_{2} C} \leq T V_{\left[x_{i_{k}}, x_{i_{k}+2[C]+1}\right]}\left(u_{h}\left(\cdot, t_{j}\right)\right) .
$$

Since we have infinite $B_{k}$, this contradicts the fact that

$$
T V_{(-\infty, \infty)}\left(u_{h}\left(\cdot, t_{j}\right)\right) \leq T V_{(-\infty, \infty)}\left(u_{0}\right)<\infty .
$$

As the trajectories of the $K+1$ waves in $A$ form a connected component, if $K<$ $2[C]+1$, there exists at least one interacting neighbor pair in $A$. Otherwise, we find the positive integer $m$ such that $m(2[C]+1)<K \leq(m+1)(2[C]+1)$, and divide $A$ into $m+1$ subsets

$$
\begin{aligned}
& B_{1}=\left\{W_{i}, \cdots, W_{i+2[C]+1}\right\}, \\
& B_{2}=\left\{W_{i+2[C]+1}, \cdots, W_{i+4[C]+2}\right\}, \\
& \cdots \\
& B_{m}=\left\{W_{i+(m-1)(2[C]+1)}, \cdots, W_{i+m(2[C]+1)}\right\}, \\
& B_{m+1}=\left\{W_{i+m(2[C]+1)}, \cdots, W_{i+K}\right\} .
\end{aligned}
$$

By Lemma 2.2, we have at least $m$ interacting neighbor pairs in $A$. We know that the total strength of discontinuities in each interacting neighboring pair is bounded below by $\frac{2 a}{c_{2} C}$ (cf.Lemma 2.1). But there is the possibility that two interacting neighbor pairs belonging to different subsets,e.g., $B_{1}, B_{2}$, share one wave. In this case, the total strength of the discontinuities in the two interacting neighbor pairs is bounded below by $\frac{2 a}{c_{2} C}$. Therefore, the total strength of discontinuities in the $m$ subsets is bounded below by $m \frac{a}{c_{2} C}$. 
Since $m \frac{a}{c_{2} C} \leq T V_{\left[x_{i_{k}}, x_{i+K}\right]}\left(u_{h}\left(\cdot, t_{j}\right)\right)$, we have

$$
m \leq \frac{c_{2} C}{a} T V_{\left[x_{i_{k}}, x_{i+K}\right]}\left(u_{h}\left(\cdot, t_{j}\right)\right) \leq \frac{c_{2} C}{a} T V_{(-\infty, \infty)}\left(u_{h}\left(\cdot, t_{j}\right)\right) .
$$

Notice that $m \geq 1$ and $T V_{(-\infty, \infty)}\left(u_{h}\left(\cdot, t_{j}\right)\right) \leq T V_{(-\infty, \infty)}\left(u_{0}\right)$,

$$
K \leq(2 m(2[C]+1)) \leq \frac{2 c_{2} C(2[C]+1)}{a} T V_{\left[x_{i_{k}}, x_{i+K}\right]}\left(u_{h}\left(\cdot, t_{j}\right)\right),
$$

therefore

$$
K \leq \frac{2 c_{2} C(2 C+1)}{a} T V_{(-\infty, \infty)}\left(\left(u_{h}\left(\cdot, t_{j}\right)\right) \leq \frac{2 c_{2} C(2 C+1)}{a} T V_{(-\infty, \infty)}\left(u_{0}\right),\right.
$$

which completes the proof.

Remark 2.5. This theorem tells us that when we use the LTS Godunov scheme to approximate general scalar conservation laws, as long as the initial data $u_{0} \in B V(\mathbb{R})$, the number of grid cells in a linear superposition zone can be bounded by the Courant number of a given problem. So when the mesh size $h$ is small, each linear superposition zone must be small.

If the flux function in (1.1) is convex, the total strength of the shocks is bounded by decreasing total variation of the initial data, thus the number of grid cells in a linear superposition zone can be bounded by the decreasing total variation of the initial data $u_{0}$, i.e.

$$
K \leq \frac{2 c_{2} C(2 C+1)}{a} D T V\left(u_{0}\right)
$$

In fact,there is at least an interacting neighboring pair in each group of $2([C]+1)$ consecutive waves if the trajectories of these waves form a connected component in the strip $t_{j}<t<t_{j+1}$ (cf.Lemma 2.2), and there is at least an interacting neighboring pair in a linear superposition zone when $K<2[C]+1$ (cf.Remark 2.3). Thus, by the inequality (2.9), $K$ can be considered as the total number of cells of all the linear superposition zones in the strip $t_{j}<t<t_{j+1}$.

\section{Estimation on the change of dissipation measure in a linear super-} position zone

In this section, we are concerned with convex scalar conservation law (1.1),(1.2) with

$$
0<c_{1} \leq f^{\prime \prime}(u) \leq c_{2}, \quad \text { for }|u| \leq M,
$$

where $c_{1}$ and $c_{2}$ are constant numbers. Denote $Q(t)$ as

$$
Q(t)=\int_{a(t)}^{b(t)} U\left(u_{h}(x, t)\right)_{t}+F\left(u_{h}(x, t)\right)_{x} d x, \quad t \in\left(t_{j}, t_{j+1}\right)
$$

where $a(t)$ and $b(t)$ are the left and right boundary of the linear superposition zone corresponding to $A$ (the trajectories of waves in $A$ form a connected component, as in Theorem 2.4). $a(t)$ is formed by all the points on the left-most waves in $A, b(t)$ is formed by all the points on the right-most waves in $A$. Evidently, they are broken lines satisfying $a(t) \geq x_{i}-\frac{C h}{\Delta t}\left(t-t_{j}\right)$ and $b(t) \leq x_{i+K}+\frac{C h}{\Delta t}\left(t-t_{j}\right)$. From Theorem 2.4, 
the length of $[a(t), b(t)]$ is of order $h$ for a given initial value problem (1.1)-(1.2). In this section, we will estimate the change of $Q(t)$ on the time interval $\left(t_{j}, t_{j}+\Delta t\right)$.

In order to investigate the interaction of a rarefaction wave with a shock, we use a fan function of a piecewise constant to approximate a rarefaction wave.

Consider a rarefaction wave

$$
R\left(x / t ; u_{l}, u_{r}\right)= \begin{cases}u_{l}, & x / t \leq f^{\prime}\left(u_{l}\right), \\ g(x / t), & f^{\prime}\left(u_{l}\right) \leq x / t \leq f^{\prime}\left(u_{r}\right), \\ u_{r}, & x / t \geq f^{\prime}\left(u_{r}\right),\end{cases}
$$

where $g$ is the inverse function of $f^{\prime}$. Denote

$$
u_{i}=u_{l}+i \frac{u_{r}-u_{l}}{n}, \quad i=0, \ldots, n,
$$

and

$$
\begin{aligned}
u_{i+1 / 2} & =\frac{1}{f^{\prime}\left(u_{i+1}\right)-f^{\prime}\left(u_{i}\right)} \int_{f^{\prime}\left(u_{i}\right)}^{f^{\prime}\left(u_{i+1}\right)} u(\xi) d \xi, \quad i=0, \ldots, n-1, \\
& =\frac{f^{\prime}\left(u_{i+1}\right) u_{i+1}-f^{\prime}\left(u_{i}\right) u_{i}-f\left(u_{i+1}\right)+f\left(u_{i}\right)}{f^{\prime}\left(u_{i+1}\right)-f^{\prime}\left(u_{i}\right)}, \\
u_{n+\frac{1}{2}} & =u_{n}, \\
A R\left(u_{l}, u_{r} ; n\right) & = \begin{cases}u_{0}=u_{l}, & x / t \leq f^{\prime}\left(u_{l}\right), \\
u_{i+1 / 2}, & f^{\prime}\left(u_{i}\right) \leq x / t \leq f^{\prime}\left(u_{i+1}\right), \quad i=0, \ldots, n, \\
u_{n}=u_{r}, & x / t \geq f^{\prime}\left(u_{r}\right) .\end{cases}
\end{aligned}
$$

Then we call $A R\left(u_{l}, u_{r} ; n\right)$ the approximate rarefaction wave for $R\left(x / t ; u_{l}, u_{r}\right)$.

In this way, we approximate a rarefaction wave by $n+1$ discontinuities with different speeds

$$
\left(u_{0}, u_{1 / 2}, f^{\prime}\left(u_{0}\right)\right),\left(u_{1 / 2}, u_{i+3 / 2}, f^{\prime}\left(u_{1}\right)\right), \cdots,\left(u_{n-1 / 2}, u_{n+\frac{1}{2}}, f^{\prime}\left(u_{n}\right)\right) .
$$

$A R\left(u_{l}, u_{r} ; n\right)$ is in fact a kind of the approximate Riemann solver proposed by Harten, Lax and Van Leer [6], which is consistent with (1.1) and satisfies the entropy condition in the sense given by them.

If there are only rarefaction waves in $\left(x_{i}, x_{i+K}\right) \times\left(t_{j}, t_{j}+\Delta t\right)$, then we have $Q(t) \equiv$ $0, t \in\left(t_{j}, t_{j}+\Delta t\right)$. When we replace $R\left(u_{l}, u_{r} ; x / t\right)$ with $A R\left(u_{l}, u_{r} ; n\right)$, this will lead to an approximation for $Q(t)$, which we call $Q_{n}(t)$. First of all, we can show that $Q_{n}(t)$ 
is negative, and will tend to zero as $n$ goes to infinity. In fact we have

$$
\begin{aligned}
Q_{n} & =\left(U\left(u_{0}\right)-U\left(u_{\frac{1}{2}}\right)\right) f^{\prime}\left(u_{0}\right)-F\left(u_{0}\right)+F\left(u_{\frac{1}{2}}\right)+ \\
& +\left(U\left(u_{\frac{1}{2}}\right)-U\left(u_{\frac{3}{2}}\right)\right) f^{\prime}\left(u_{1}\right)-F\left(u_{\frac{1}{2}}\right)+F\left(u_{\frac{3}{2}}\right)+ \\
& +\cdots+ \\
& +\left(U\left(u_{i-\frac{1}{2}}\right)-U\left(u_{i+\frac{1}{2}}\right)\right) f^{\prime}\left(u_{i}\right)-F\left(u_{i-\frac{1}{2}}\right)+F\left(u_{i+\frac{1}{2}}\right)+ \\
& +\cdots+ \\
& +\left(U\left(u_{n-\frac{1}{2}}\right)-U\left(u_{n}\right)\right) f^{\prime}\left(u_{n}\right)-F\left(u_{n-\frac{1}{2}}\right)+F\left(u_{n}\right) \\
& =U\left(u_{0}\right) f^{\prime}\left(u_{0}\right)+U\left(u_{\frac{1}{2}}\right)\left(f^{\prime}\left(u_{1}\right)-f^{\prime}\left(u_{0}\right)\right)+ \\
& +\cdots+ \\
& +U\left(u_{i+\frac{1}{2}}\right)\left(f^{\prime}\left(u_{i+1}\right)-f^{\prime}\left(u_{i}\right)\right)+ \\
& +\cdots+ \\
& +U\left(u_{n-\frac{1}{2}}\right)\left(f^{\prime}\left(u_{n}\right)-f^{\prime}\left(u_{n-1}\right)\right)- \\
& -U\left(u_{n}\right) f^{\prime}\left(u_{n}\right)-F\left(u_{0}\right)+F\left(u_{n}\right)
\end{aligned}
$$

By Taylor expansion, we have

$$
U(u(\xi))=U\left(u_{i+\frac{1}{2}}\right)-U^{\prime}\left(u_{i+\frac{1}{2}}\right)\left(u_{i+\frac{1}{2}}-u(\xi)\right)+\frac{1}{2} U^{\prime \prime}(\tilde{u})\left(u(\xi)-u_{i+\frac{1}{2}}\right)^{2} .
$$

If we set $\eta=u(\xi)$, then $f^{\prime}(u(\xi))=\xi, f^{\prime}(\eta)=\xi, d \xi=f^{\prime \prime}(\eta) d \eta$. From the definition of $u_{i+\frac{1}{2}}$, we have

$$
\int_{f^{\prime}\left(u_{i}\right)}^{f^{\prime}\left(u_{i+1}\right)}\left(u_{i+\frac{1}{2}}-u(\xi)\right) d \xi=0
$$

Therefore,

$$
\begin{aligned}
& \frac{1}{f^{\prime}\left(u_{i+1}\right)-f^{\prime}\left(u_{i}\right)} \int_{f^{\prime}\left(u_{i}\right)}^{f^{\prime}\left(u_{i+1}\right)} U(u(\xi)) d \xi \\
&= U\left(u_{i+\frac{1}{2}}\right)+\frac{1}{2\left(f^{\prime}\left(u_{i+1}\right)-f^{\prime}\left(u_{i}\right)\right)} \int_{f^{\prime}\left(u_{i}\right)}^{f^{\prime}\left(u_{i+1}\right)} U^{\prime \prime}(\tilde{u})\left(u(\xi)-u_{i+\frac{1}{2}}\right)^{2} d \xi \\
&= U\left(u_{i+\frac{1}{2}}\right)+\frac{U^{\prime \prime}(\hat{u})}{2\left(f^{\prime}\left(u_{i+1}\right)-f^{\prime}\left(u_{i}\right)\right)} \int_{u_{i}}^{u_{i+1}} f^{\prime \prime}(\eta)\left(\eta-u_{i+\frac{1}{2}}\right)^{2} d \eta \\
& U\left(u_{i+\frac{1}{2}}\right)+\frac{U^{\prime \prime}(\tilde{u}) f^{\prime \prime}(\hat{\eta})}{2\left(f^{\prime}\left(u_{i+1}\right)-f^{\prime}\left(u_{i}\right)\right)} \frac{u_{i+1}-u_{i}}{3}\left[\left(u_{i+1}-u_{i+\frac{1}{2}}\right)^{2}+\right. \\
&\left.\quad+\left(u_{i+1}-u_{i+\frac{1}{2}}\right)\left(u_{i}-u_{i+\frac{1}{2}}\right)+\left(u_{i}-u_{i+\frac{1}{2}}\right)^{2}\right] \\
&=U\left(u_{i+\frac{1}{2}}\right)+\frac{U^{\prime \prime}(\tilde{u}) f^{\prime \prime}(\hat{\eta})}{6 f^{\prime \prime}(\tilde{\eta})}\left[\left(u_{i+1}-u_{i+\frac{1}{2}}\right)^{2}+\right. \\
&\left.+\left(u_{i+1}-u_{i+\frac{1}{2}}\right)\left(u_{i}-u_{i+\frac{1}{2}}\right)+\left(u_{i}-u_{i+\frac{1}{2}}\right)^{2}\right] .
\end{aligned}
$$

Since

$$
0>\left(u_{i+1}-u_{i+\frac{1}{2}}\right)\left(u_{i}-u_{i+\frac{1}{2}}\right) \geq-\frac{\left(u_{i+1}-u_{i+\frac{1}{2}}\right)^{2}+\left(u_{i}-u_{i+\frac{1}{2}}\right)^{2}}{2}
$$


and

$$
0<c_{1} \leq f^{\prime \prime}(u) \leq c_{2}, \quad 0<c_{a} \leq U^{\prime \prime}(u) \leq c_{b},
$$

we have

$$
\begin{array}{r}
\frac{1}{f^{\prime}\left(u_{i}\right)-f^{\prime}\left(u_{i+1}\right)} \int_{f^{\prime}\left(u_{i}\right)}^{f^{\prime}\left(u_{i+1}\right)} U(u(\xi)) d \xi \geq U\left(u_{i+\frac{1}{2}}\right) \\
+\frac{c_{a} c_{1}}{12 c_{2}}\left[\left(u_{i+1}-u_{i+\frac{1}{2}}\right)^{2}+\left(u_{i}-u_{i+\frac{1}{2}}\right)^{2}\right] .
\end{array}
$$

Moreover,

$$
\begin{aligned}
u_{i+1}-u_{i+\frac{1}{2}} & =\frac{1}{f^{\prime}\left(u_{i+1}\right)-f^{\prime}\left(u_{i}\right)} \int_{f^{\prime}\left(u_{i}\right)}^{f^{\prime}\left(u_{i+1}\right)}\left(u_{i+1}-u(\xi)\right) d \xi \\
& =\frac{1}{f^{\prime}\left(u_{i+1}\right)-f^{\prime}\left(u_{i}\right)} \int_{u_{i}}^{u_{i+1}}\left(u_{i+1}-\eta\right) f^{\prime \prime}(\eta) d \eta \\
& =\frac{f^{\prime \prime}(\hat{\eta})}{f^{\prime}\left(u_{i+1}\right)-f^{\prime}\left(u_{i}\right)} \cdot \frac{1}{2}\left(u_{i+1}-u_{i}\right)^{2} \\
& \geq \frac{c_{1}}{2 c_{2}}\left(u_{i+1}-u_{i}\right),
\end{aligned}
$$

Finally, we have

$$
\frac{1}{f^{\prime}\left(u_{i+1}\right)-f^{\prime}\left(u_{i}\right)} \int_{f^{\prime}\left(u_{i}\right)}^{f^{\prime}\left(u_{i+1}\right)} U(u(\xi)) d \xi \geq U\left(u_{i+\frac{1}{2}}\right)+\frac{c_{a} c_{1}^{3}}{24 c_{2}^{3}}\left(u_{i+1}-u_{i}\right)^{2} .
$$

Hence,

$$
U\left(u_{i+\frac{1}{2}}\right)\left(f^{\prime}\left(u_{i+1}\right)-f^{\prime}\left(u_{i}\right)\right) \leq \int_{f^{\prime}\left(u_{i}\right)}^{f^{\prime}\left(u_{i+1}\right)} U(u(\xi)) d \xi-\frac{c_{a} c_{1}^{4}}{24 c_{2}^{3}}\left(u_{i+1}-u_{i}\right)^{3} .
$$

Using the inequality (3.4) in (3.2) gives

$$
\begin{aligned}
& Q_{n} \leq \int_{f^{\prime}\left(u_{r}\right)}^{f^{\prime}\left(u_{l}\right)} U(u(\xi)) d \xi-U\left(u_{r}\right) f^{\prime}\left(u_{r}\right)+U\left(u_{l}\right) f^{\prime}\left(u_{l}\right)-F\left(u_{l}\right)+F\left(u_{r}\right)- \\
& \quad-\frac{c_{a} c_{1}^{4}}{24 c_{2}^{3}} \sum_{i=0}^{n-1}\left(u_{i+1}-u_{i}\right)^{3} .
\end{aligned}
$$

Since $u(\xi)$ is a rarefaction wave, we have

$$
\int_{f^{\prime}\left(u_{r}\right)}^{f^{\prime}\left(u_{l}\right)} U(u(\xi)) d \xi-U\left(u_{r}\right) f^{\prime}\left(u_{r}\right)+U\left(u_{l}\right) f^{\prime}\left(u_{l}\right)-F\left(u_{l}\right)+F\left(u_{r}\right)=0 .
$$

Noting that $u_{i+1}-u_{i}=\frac{u_{r}-u_{l}}{n}$, we obtain

$$
Q_{n} \leq-\frac{c_{a} c_{1}^{4}}{24 c_{2}^{3}} \frac{\left(u_{r}-u_{l}\right)^{3}}{n^{2}}<0
$$

Now we estimate the change of $Q(t)$ when two discontinuities interact linearly with each other. 


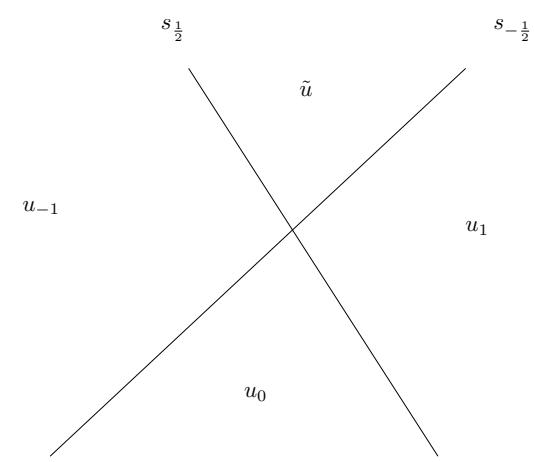

FIG. 3.1. A pair of interacted discontinuities

Lemma 3.1. Suppose that a pair of neighboring discontinuities $\left(u_{-1}, u_{0}, s_{-\frac{1}{2}}\right)$ and $\left(u_{0}, u_{1}, s_{\frac{1}{2}}\right)$ interact linearly with each other(cf. figure 3.1). Then, through the interaction, $\stackrel{Q}{Q}(t)$ must be decreased when (i) $u_{-1}>u_{0}>u_{1}$, or (ii) $u_{-1}<u_{0}<u_{1}$; must be increased when (iii) $u_{-1}<u_{0}$ and $u_{0}>u_{1}$, or (iv) $u_{-1}>u_{0}$ and $u_{0}<u_{1}$.

Proof: Before the interaction,

$$
\begin{array}{r}
Q_{1}=\left(U\left(u_{-1}\right)-U\left(u_{0}\right)\right) s_{-\frac{1}{2}}-F\left(u_{-1}\right)+F\left(u_{0}\right)+ \\
+\left(U\left(u_{0}\right)-U\left(u_{1}\right)\right) s_{\frac{1}{2}}-F\left(u_{0}\right)+F\left(u_{1}\right) .
\end{array}
$$

After the interaction,

$$
\begin{aligned}
Q_{2}=( & \left.\left(u_{-1}\right)-U(\tilde{u})\right) s_{\frac{1}{2}}-F\left(u_{-1}\right)+F(\tilde{u})+ \\
& +\left(U(\tilde{u})-U\left(u_{1}\right)\right) s_{-\frac{1}{2}}-F(\tilde{u})+F\left(u_{1}\right),
\end{aligned}
$$

where $\tilde{u}=u_{-1}+u_{1}-u_{0}$.

We study their difference

$$
\begin{aligned}
-\left(Q_{1}-Q_{2}\right) & =-\left(U\left(u_{-1}\right)-U(\tilde{u})-U\left(u_{0}\right)+U\left(u_{1}\right)\right)\left(s_{-\frac{1}{2}}-s_{\frac{1}{2}}\right) \\
& =-\int_{u_{0}}^{u_{-1}} \int_{u+\left(u_{1}-u_{0}\right)}^{u} U^{\prime \prime}(\eta) d \eta d u \cdot\left(s_{-\frac{1}{2}}-s_{\frac{1}{2}}\right) .
\end{aligned}
$$

In order to make sure that the two discontinuities interact with each other, we must have $s_{\frac{1}{2}}<s_{-\frac{1}{2}}$. Thus,

$$
\begin{aligned}
-\operatorname{sign}\left(Q_{1}-Q_{2}\right) & =\operatorname{sign}\left(\int_{u_{0}}^{u_{-1}} \int_{u+\left(u_{1}-u_{0}\right)}^{u} U^{\prime \prime}(\eta) d \eta d u\right) \\
& =-\operatorname{sign}\left(\left(u_{-1}-u_{0}\right)\left(u_{1}-u_{0}\right)\right) .
\end{aligned}
$$

Here $U^{\prime \prime}>0$ is used. And the conclusion of the lemma follows from (3.7).

Now, we consider a typical case for the linear interactions in a LTS scheme. Suppose that $A R\left(u_{-1}, u_{0} ; n\right)$ and $S\left(\tilde{u}_{0}, u_{1}\right)$ are in the same linear superposition zone $D$, and they interact with each other in $D$. We denote the total strength of all the shocks between $A R\left(u_{-1}, u_{0} ; n\right)$ and $S\left(\tilde{u}_{0}, u_{1}\right)$ by $\bar{S}$, cf. figure 3.2 . 


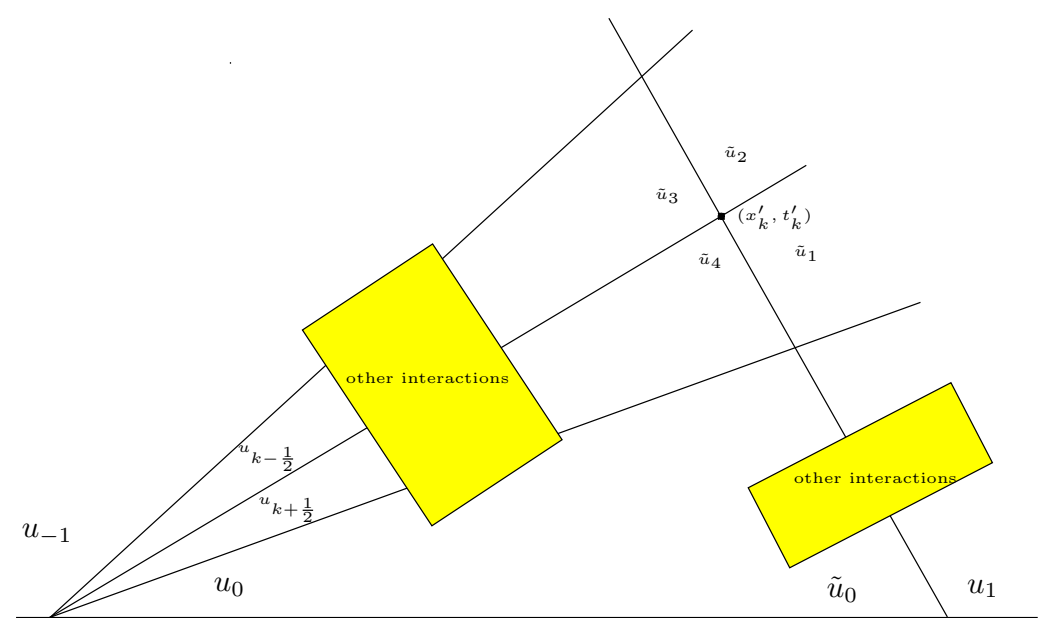

FIG. 3.2. Figure for Lemma 3.2

Lemma 3.2. For the typical case mentioned above, $Q_{n}$ will increase after the interaction of $A R\left(u_{-1}, u_{0} ; n\right)$ and $S\left(\tilde{u}_{0}, u_{1}\right)$, and the increased amount $\Delta Q_{n}$ satisfies

$$
\Delta Q_{n} \leq \frac{n+1}{n} \frac{c_{2} c_{b}}{2}\left(u_{0}-u_{-1}\right)\left(\tilde{u}_{0}-u_{1}\right)\left[2 \bar{S}+\left(\tilde{u}_{0}-u_{1}\right)\right]
$$

Proof: $Q_{n}(t)$ remains unchanged until the interaction. Consider the interaction point $\left(x_{k}^{\prime}, t_{k}^{\prime}\right)$ of a discontinuity in $A R\left(u_{-1}, u_{0} ; n\right)$

$$
x-x_{i}=f^{\prime}\left(u_{k}^{\prime}\right)\left(t-t_{j}\right),
$$

with the shock

$$
x-x_{i+1}=\frac{f\left(\tilde{u}_{0}\right)-f\left(u_{1}\right)}{\tilde{u}_{0}-u_{1}}\left(t-t_{j}\right) .
$$

From (3.6), we have

$$
\begin{aligned}
& Q\left(t_{k}^{\prime}+0\right)-Q\left(t_{k}^{\prime}-0\right) \\
& =\int_{\tilde{u}_{4}}^{\tilde{u}_{3}} \int_{u+\left(\tilde{u}_{1}-\tilde{u}_{4}\right)}^{u} U^{\prime \prime}(\eta) d \eta\left(\frac{f\left(\tilde{u}_{0}\right)-f\left(u_{1}\right)}{\tilde{u}_{0}-u_{1}}-f^{\prime}\left(u_{k}^{\prime}\right)\right) d u \\
& \leq \int_{\tilde{u}_{3}}^{\tilde{u}_{4}} \int_{u+\left(\tilde{u}_{1}-\tilde{u}_{4}\right)}^{u} U^{\prime \prime}(\eta) d \eta\left(f^{\prime}\left(u_{0}\right)-\frac{f\left(\tilde{u}_{0}\right)-f\left(u_{1}\right)}{\tilde{u}_{0}-u_{1}}\right) d u .
\end{aligned}
$$


Since

$$
\begin{aligned}
& \left(f^{\prime}\left(u_{0}\right)-\frac{f\left(\tilde{u}_{0}\right)-f\left(u_{1}\right)}{\tilde{u}_{0}-u_{1}}\right) \\
& =\int_{0}^{1}\left(f^{\prime}\left(u_{0}\right)-f^{\prime}\left(\tilde{u}_{0} \xi+u_{1}(1-\xi)\right)\right) d \xi \\
& =\int_{0}^{1} f^{\prime \prime}(\eta)\left[\left(u_{0}-\tilde{u}_{0}\right) \xi+\left(u_{0}-u_{1}\right)(1-\xi)\right] d \xi \\
& \leq \frac{c_{2}}{2}\left[\left(u_{0}-\tilde{u}_{0}\right)+\left(u_{0}-u_{1}\right)\right] \\
& \leq \frac{c_{2}}{2}\left[2 \sum_{j}\left|S_{j}\right|+\left(\tilde{u}_{0}-u_{1}\right)\right]
\end{aligned}
$$

where $S_{j}^{\prime} s$ are the shocks between $A R\left(u_{-1}, u_{0} ; n\right)$ and $S\left(\tilde{u}_{0}, u_{1}\right),\left|S_{j}\right|$ is the strength of $S_{j}$. So

$$
Q\left(t_{k}^{\prime}+0\right)-Q\left(t_{k}^{\prime}-0\right) \leq \frac{c_{2} c_{b}}{2}\left(\tilde{u}_{4}-\tilde{u}_{1}\right)\left(\tilde{u}_{4}-\tilde{u}_{3}\right)\left[2 \sum_{j}\left|S_{j}\right|+\left(\tilde{u}_{0}-u_{1}\right)\right] .
$$

Noting that

$$
\tilde{u}_{4}-\tilde{u}_{3}=u_{k+\frac{1}{2}}-u_{k-\frac{1}{2}}, \quad \tilde{u}_{4}-\tilde{u}_{1}=\tilde{u}_{0}-u_{1}
$$

and using the definition of $u_{k+\frac{1}{2}}$, we have

$$
Q\left(t_{k}^{\prime}+0\right)-Q\left(t_{k}^{\prime}-0\right) \leq \frac{c_{2} c_{b}}{2} \frac{u_{0}-u_{-1}}{n}\left(\tilde{u}_{0}-u_{1}\right)\left[2 \sum_{j}\left|S_{j}\right|+\left(\tilde{u}_{0}-u_{1}\right)\right]
$$

Summing with respect to $k$, we get the total increased amount:

$$
\begin{aligned}
\Delta Q_{n} & =\sum_{k}\left(Q\left(t_{k}^{\prime}+0\right)-Q\left(t_{k}^{\prime}-0\right)\right) \\
& \leq \frac{c_{2} c_{b}}{2} \frac{n+1}{n}\left(u_{0}-u_{-1}\right)\left(\tilde{u}_{0}-u_{1}\right)\left[2 \sum_{j}\left|S_{j}\right|+\left(\tilde{u}_{0}-u_{1}\right)\right]
\end{aligned}
$$

which completes the proof.

Now we need an inequality (3.9) which can be deduced from the Hölder inequality to estimate $Q_{n}(t)$ in a linear superposition zone.

Assume that $a_{1}, a_{2}, \ldots, a_{n}$ are positive real numbers. Then we have

$$
n^{2} \sum_{i=1}^{n} a_{i}^{3} \geq\left(\sum_{i=1}^{n} a_{i}\right)^{3}
$$

Lemma 3.3. Assume that there are $l$ shocks $S_{1}, S_{2}, \ldots, S_{l}$ and $m(m \geq 1)$ approximate rarefaction waves $A R_{1}, A R_{2}, \ldots, A R_{m}$ in a linear superposition zone $D$, with

$$
S=\sum_{i=1}^{l}\left|S_{i}\right|, \quad R=\sum_{j=1}^{m}\left|R_{j}\right|
$$


Then for any $t \in\left(t_{j}, t_{j}+\Delta t\right)$,

$$
Q_{n}(t) \leq \frac{c_{2} c_{b}}{2} S^{2}\left[\frac{2(n+1)}{n} R-\frac{c_{1} c_{a}}{c_{2} c_{b}} l^{-2} S\right]
$$

where $\left|R_{j}\right|$ is the strength of $A R_{j}$.

Proof: For $t \in\left(t_{j}, t_{j}+\Delta t\right), Q_{n}(t)$ remains unchanged until the interaction happens. By Lemma 3.1, $Q_{n}(t)$ increases only when it passes a interaction point of a shock and a discontinuity in an approximate rarefaction wave. From Lemma 3.2, for a typical case $\left(x_{k}^{\prime}, t_{k}^{\prime}\right)$ we have

$$
\Delta Q_{n} \leq c_{2} c_{b} \frac{n+1}{n}\left(u_{0}-u_{-1}\right)\left(\tilde{u}_{0}-u_{1}\right) S
$$

Summing all the interaction points from $t_{j}$ to $t$, we have,

$$
Q_{n}(t)-Q\left(t_{j}+0\right) \leq c_{2} c_{b} \frac{n+1}{n} S^{2} R
$$

where

$$
Q\left(t_{j}+0\right) \leq-\frac{c_{1} c_{a}}{2} \sum_{i=1}^{l}\left|S_{i}\right|^{3}
$$

From (3.9)

$$
\begin{aligned}
Q_{n}(t) & \leq \frac{c_{2} c_{b}}{2}\left[\frac{2(n+1)}{n} S^{2} R-\frac{c_{1} c_{a}}{c_{2} c_{b}} \sum_{i=1}^{l}\left|S_{i}\right|^{3}\right] \\
& \leq \frac{c_{2} c_{b}}{2}\left[\frac{2(n+1)}{n} S^{2} R-\frac{c_{1} c_{a}}{c_{2} c_{b}} l^{-2} S^{3}\right]
\end{aligned}
$$

which completes the proof.

Lemma 3.4. Assume that there are $l$ shocks $S_{1}, S_{2}, \ldots, S_{l}$ and $m$ rarefaction waves $R_{1}, R_{2}, \ldots, R_{m}$ in a linear superposition zone $D$. Denote $R$ as the total strength of $R_{1}, R_{2}, \ldots, R_{m}, S$ as the total strength of $S_{1}, S_{2}, \ldots, S_{l}$, and

$$
\left.<\eta, 1>\left.\right|_{D}=\int_{t_{j}}^{t_{j+1}} \int_{a(t)}^{b(t)}\left(U\left(u_{h}\right)_{t}\right)+F\left(u_{h}\right)_{x}\right) d x d t .
$$

Then

$$
<\eta, 1>\left.\right|_{D} \leq\left(1-\frac{1}{2 C}\right) \Delta t c_{2} c_{b} S^{2}\left[R-\frac{c_{1} c_{a}}{c_{2} c_{b}} \frac{C}{2 C-1} l^{-2} S\right]
$$

Proof: Denote $u_{n}$ as the linear superposition of $l$ shocks $S_{1}, S_{2}, \ldots, S_{l}$ and $m$ approximate rarefaction waves $A R_{1}, A R_{2}, \ldots, A R_{m}$ ( $u_{n}$ is a approximation to $u_{h}$ ), and denote

$$
\eta_{n}=U\left(u_{n}\right)_{t}+F\left(u_{n}\right)_{x}
$$


Then, as any pair of waves from $\left(x_{i}, t_{j}\right)$ and $\left(x_{k}, t_{j}\right)$ cannot meet each other before

$$
t=t_{j}+\frac{\Delta t}{2 C}
$$

by (3.9), (3.5) and a classical result for shock (see Lemma 2.1 in [16]), we have

$$
\begin{aligned}
<\eta_{n}, 1>\left.\right|_{D} \leq & \int_{t_{j}+\frac{\Delta t}{2 C}}^{t_{j+1}} \int_{a(t)}^{b(t)} \eta_{n} d x d t+\int_{t_{j}}^{t_{j}+\frac{\Delta t}{2 C}} \int_{a(t)}^{b(t)} \eta_{n} d x d t \\
\leq & \int_{t_{j}+\frac{\Delta t}{2 C}}^{t_{j+1}} Q_{n}(t) d t+\int_{t_{j}}^{t_{j}+\frac{\Delta t}{2 C}}\left(-\frac{c_{1} c_{a}}{2} \sum_{i=1}^{l}\left|S_{i}\right|^{3}\right) d t \\
\leq & \Delta t\left(1-\frac{1}{2 C}\right) \frac{c_{2} c_{b}}{2} S^{2}\left[\frac{2(n+1)}{n} R-\frac{c_{1} c_{a}}{c_{2} c_{b}} l^{-2} S\right] \\
& \quad-\frac{\Delta t}{2 C} \frac{c_{1} c_{a}}{2} l^{-2} S^{3} \\
\leq & \Delta t\left(1-\frac{1}{2 C}\right) c_{2} c_{b} S^{2}\left[\frac{n+1}{n} R-\frac{c_{1} c_{a}}{c_{2} c_{b}} \frac{C}{2 C-1} l^{-2} S\right] .
\end{aligned}
$$

Since $A R\left(u_{l}, u_{r} ; n\right)$ converges to $R\left(u_{l}, u_{r} ; x / t\right)$ point-wisely when $n \longrightarrow \infty$, we have

$$
\begin{aligned}
<\eta, 1>\left.\right|_{D} & =\lim _{n \rightarrow \infty}<\eta_{n}, 1>\left.\right|_{D} \\
& \leq\left(1-\frac{1}{2 C}\right) \Delta t c_{2} c_{b} S^{2}\left[R-\frac{c_{1} c_{a}}{c_{2} c_{b}} \frac{C}{2 C-1} l^{-2} S\right]
\end{aligned}
$$

which completes the proof.

\section{Entropy consistency of the LTS Godunov scheme}

In this section, we will prove an entropy consistency theorem on the LTS Godunov scheme. It is well known (cf. [4]) that for convex scalar conservation laws a weak solution satisfies (1.5) for all convex entropy pairs $\{U(u), F(u)\}$ if it satisfies (1.5) for the special entropy pair

$$
U(u)=\frac{u^{2}}{2}, \quad F(u)=\int^{u} u f^{\prime}(u) d u .
$$

Theorem 4.1. Assume that the initial data $u_{0}(x)$ satisfies (1.3). For the LTS Godunov scheme, a sufficient condition to ensure the entropy consistency for any given Courant number $C$ is

$$
\operatorname{ITV}\left(u_{0}\right) \operatorname{DTV}\left(u_{0}\right) \leq \frac{a^{2} c_{1}}{4 c_{2}^{3} C(2 C-1)(2 C+1)^{2}} .
$$

Proof: By Theorem 2.4, in a linear superposition zone $D$, there are at most $K+1$ waves, and

$$
K \leq \frac{2 c_{2} C(2 C+1)}{a} D T V\left(u_{0}\right)
$$

If all of them are shocks, by Lemma 3.1, we have $<\eta, 1>\left.\right|_{D}<0$. Otherwise, there are at most $K$ shocks among the $K+1$ waves. By Lemma 2.1 and Lemma 2.2, there are 
at least $m$ shocks $\left(m \geq \frac{K}{2(2[C]+1)}\right)$ in $D$ with total strength of all these shocks bounded below by $\frac{m a}{c_{2} C}$. By Lemma 3.4, we have $<\eta, 1>\left.\right|_{D} \leq 0$ if

$$
\Delta=\sum_{i}\left|R_{i}\right|-\frac{c_{1}}{K^{2} c_{2}} \frac{C}{2 C-1} \sum_{j}\left|S_{j}\right| \leq 0 .
$$

Since

$$
\sum_{R_{i} \in D}\left|R_{i}\right| \leq I T V\left(u_{0}\right), \quad \sum_{S_{j} \in D}\left|S_{j}\right| \geq m \frac{a}{c_{2} C}
$$

when $\operatorname{DTV}\left(u_{0}\right) \neq 0$, we have

$$
\begin{aligned}
\Delta & \leq I T V\left(u_{0}\right)-\frac{c_{1}}{c_{2}^{2}} \frac{1}{K^{2}} \frac{a}{2 C-1} m \\
& \leq I T V\left(u_{0}\right)-\frac{a c_{1}}{c_{2}^{2}} \frac{1}{K} \frac{1}{2 C-1} \frac{1}{2(2[C]+1)} \\
& \leq \operatorname{ITV}\left(u_{0}\right)-\frac{a^{2} c_{1}}{4 c_{2}^{3}} \frac{1}{C(2 C-1)(2 C+1)^{2}} \frac{1}{D T V\left(u_{0}\right)} \\
& \leq 0
\end{aligned}
$$

where we have used (4.2) in the final inequality. If $D T V\left(u_{0}\right)=0$, the initial data is monotone increasing, there are only rarefaction waves in the solution and there is no interaction, so the entropy consistency can be guaranteed.

Finally, consider the relation between the inequality

$$
<\eta, 1>\left.\right|_{D} \leq 0
$$

and the cell entropy inequality

$$
\begin{aligned}
& \int_{x_{i}}^{x_{i+1}} U\left(u\left(x, t_{j+1}+0\right) d x-\right. \\
& -\int_{x_{i}}^{x_{i+1}} U\left(u\left(x, t_{j}+0\right) d x+\int_{t_{i}}^{t_{i+1}}\left(F \left(u\left(x_{i+1}, t\right)-F\left(u\left(x_{i}, t\right)\right) d t \leq 0\right.\right.\right.
\end{aligned}
$$

where $\{U, F\}$ is the entropy pair (4.1).

By the generalized Gauss-Green formula for $B V$ functions [5], on any cell $D_{i j}=$ $\left(x_{i}, x_{i+1}\right) \times\left(t_{j}, t_{j+1}\right)$,

$$
\begin{array}{rl}
<\eta, 1>\left.\right|_{D_{i j}} & \\
=\int_{x_{i}}^{x_{i+1}} & U\left(u\left(x, t_{j+1}-0\right) d x-\int_{x_{i}}^{x_{i+1}} U\left(u\left(x, t_{j}+0\right) d x\right.\right. \\
& +\int_{t_{i}}^{t_{i+1}}\left(F \left(u\left(x_{i+1}, t\right)-F\left(u\left(x_{i}, t\right)\right) d t,\right.\right.
\end{array}
$$

thus

$$
\begin{aligned}
& \int_{x_{i}}^{x_{i+1}} U\left(u\left(x, t_{j+1}+0\right) d x-\right. \\
& -\int_{x_{i}}^{x_{i+1}} U\left(u\left(x, t_{j}+0\right) d x+\int_{t_{i}}^{t_{i+1}}\left(F \left(u\left(x_{i+1}, t\right)-F\left(u\left(x_{i}, t\right)\right) d t\right.\right.\right. \\
& =<\eta, 1>\left.\right|_{D_{i j}}+\left[\int _ { x _ { i } } ^ { x _ { i + 1 } } U \left(u\left(x, t_{j+1}+0\right) d x-\int_{x_{i}}^{x_{i+1}} U\left(u\left(x, t_{j+1}-0\right) d x\right] .\right.\right.
\end{aligned}
$$


As the second term in the right hand side is non-positive from Jensen's inequality and the construction of Godunov scheme, we can get the ordinary cell entropy inequality when $<\eta, 1>\left.\right|_{D_{i j}} \leq 0$. In the LTS Godunov scheme with the Courant number $C>1$, if there is no interaction happening in $D$, or $D$ is a linear superposition zone, (4.3) is valid. From Theorem 2.4, the number of waves in a linear superposition zone is finite and independent of the mesh size $h$, so we can add the neighboring cells, in which there are no interactions of waves, to one or several linear superposition zones, and form a rectangular zone $D_{i}$,

$$
D_{i}=\left\{(x, t) \mid x_{i} \leq x<x_{i+k_{i}}, t_{j} \leq t<t_{j+1}\right\}
$$

By

$$
\int_{t_{i}}^{t_{i+1}} F\left(u\left(x_{i}-0, t\right)\right) d t=\int_{t_{i}}^{t_{i+1}} F\left(u\left(x_{i}+0, t\right)\right) d t
$$

we have

$$
\begin{aligned}
& \int_{x_{i}}^{x_{i+k_{i}}} U\left(u\left(x, t_{j+1}+0\right) d x-\right. \\
& -\int_{x_{i}}^{x_{i+k_{i}}} U\left(u\left(x, t_{j}+0\right) d x+\int_{t_{i}}^{t_{i+1}}\left(F \left(u\left(x_{i+k_{i}}, t\right)-F\left(u\left(x_{i}, t\right)\right) d t\right.\right.\right. \\
& =<\eta, 1>\left.\right|_{D_{i}}+\left[\int _ { x _ { i } } ^ { x _ { i + 1 } } U \left(u\left(x, t_{j+1}+0\right) d x-\int_{x_{i}}^{x_{i+1}} U\left(u\left(x, t_{j+1}-0\right) d x\right]\right.\right. \\
& +\left[\int _ { x _ { i + 1 } } ^ { x _ { i + 2 } } U \left(u\left(x, t_{j+1}+0\right) d x-\int_{x_{i+1}}^{x_{i+2}} U\left(u\left(x, t_{j+1}-0\right) d x\right]\right.\right. \\
& \cdots \ldots \\
& +\left[\int _ { x _ { i + k _ { i } - 1 } } ^ { x _ { i + k _ { i } } } U \left(u\left(x, t_{j+1}+0\right) d x-\int_{x_{i+k_{i}-1}}^{x_{i+k_{i}}} U\left(u\left(x, t_{j+1}-0\right) d x\right]\right.\right. \\
& \leq<\eta, 1>\left.\right|_{D_{i}} \leq 0 .
\end{aligned}
$$

This is in fact a cell entropy inequality on $D_{i}$. By the technique used in the LaxWendroff Theorem[10], this inequality also implies the entropy condition (1.5) for the LTS Godunov scheme.

Acknowledgements. We appreciate professor Tao Tang for helping us to improve the presentation of this paper. The first author would like to express deep thanks to professor Gerald Warnecke for generous discussions on the various LTS schemes.

\section{REFERENCES}

[1] Y. Brenier, Averaged multivalued solutions for scalar conservation laws, SIAM J. Numer. Anal., 21, 1013-1037, 1984.

[2] Y. Brenier, Stability du schema de Glimm Roe pour les systems de lois de conservatiom, Rapports de Recherche, INRIA, Rocquencourt, 1985.

[3] R. DiPerna, Entropy and uniqueness of solutions to hyperbolic conservation laws, Nonlinear Evolution Equations, Academic Press, New York, 1-15, 1978.

[4] R. DiPerna, Convergence of approximate solutions to conservation laws, Arch. Rat. Mech. Anal., Vol. 82, 27-70, 1983. 
[5] L.C. Evans and R.F. Gariepy, Measure Theory and Fine Properties of Functions, CRC Press, Inc., 1992.

[6] A. Harten, P.D. Lax, B. and van Leer, On upstream differencing and Godunov-type schemes for hyperbolic conservation laws, SIAM Review, 25, 35-61, 1983.

[7] J. Huang, J. Wang, and G. Warnecke, Error bounds for the large time step Glimm scheme applied to scalar conservation laws, Numer. Math., 91, 13-34, 2002.

[8] C. Helzel and G. Warnecke, Unconditionally stable explicit schemes approximation for conservation laws, Ergodic Theory, Analysis and Efficient Simulation of Dynamical Systems (Ed.by B.Fiedler), Springer-Verlag, Berlin, 775-803, 2001.

[9] G.-S. Jiang, and J. Wang, Entropy consistency of large time step schemes for isentropic equations of gas dynamics, Acta Math. Sci., 13 (4), 361-383, 1993.

[10] P.D. Lax and B. Wendroff, Systems of conservation laws, Comm. Pure Appl. Math., 13, 217237,1960

[11] R.J. LeVeque, Convergence of a large time generalization of Godunov's method for conservation laws, Comm. Pure and Appl. Math., 37 (6), 463-477, 1984.

[12] R.J. LeVeque, A large time generalization of Godunov's method for conservation laws, SIAM J. Numer. Anal., 22 (6), 1051-1073, 1985.

[13] B. Lucier, Error bounds for the methods of Glimm, Godunov and LeVeque, SIAM J. Numer. Anal., 22, 1074-1081, 1985.

[14] E.F. Toro and S.J. Billett, A unified Riemann-problem-based extension of the Warming-Beam and Lax-Wendroff schemes, IMA J. Numer. Anal., 17, 61-102, 1997.

[15] J. Wang, Large time step generalizations of Glimm's scheme for systems of conservation laws, Chin. Ann. of Math., 9B, 54-63, 1988.

[16] J. Wang and G. Warnecke, On entropy consistency of large time step schemes I. the Godunov and Glimm scheme, SIAM J. Numer. Anal., 30 (5), 1229-1251, 1993.

[17] J. Wang and G. Warnecke, On entropy consistency of large time step schemes II. approximate Riemann solvers, SIAM J. Numer. Anal., 30 (5), 1252-1267, 1993. 